\title{
On the dispersion characteristics of slow waves supported by the waveguide configuration of a dielectric sandwiched between two plasma slabs
}

\author{
Essam Yasin ${ }^{1}$, Ahmed AlKhateeb ${ }^{2}$, Fuad Rawwagah ${ }^{2}$, \\ Alaaedeen Abuzor ${ }^{1}$ \\ ${ }^{1}$ Department of Physics, King Faisal University, Alhasa, Saudi Arabia \\ ${ }^{2}$ Department of Physics, Yarmouk University, Irbid, Jordan \\ *corresponding author, E-mail: eyasinekfu.edu.sa
}

\begin{abstract}
We derived a general equation governing the spectra of electrostatic surface plasmons supported by a waveguide structure of two identical plasma slabs separated by a dielectric medium. The plasma slabs are parallel, homogeneous, and have finite thicknesses. The geometry under consideration supports two surface plasmon modes, which we investigated numerically for Polyethylene $\varepsilon_{d}=2.25$ and vacuum $\varepsilon_{d}=1$ as central regions. With vacuum as a central region, the two surface plasmon modes become coupled and merge into the well known single mode of quasi-static frequency $\omega=0.707 \omega_{\mathrm{p}}$. The surface plasmon modes in the presence of a Polyethylene are decoupled and remain nondegenerate over the whole range of $k d$. Therefore, the two plasmon modes propagate independent of each other with distinct quasi-static resonance frequencies, namely, a backward wave with $\omega=0.707 \omega_{\mathrm{p}}$ corresponding to a single plasma-vacuum interface and a forward wave with $\omega=\frac{\omega_{\mathrm{p}}}{\sqrt{3.25}}=0.55 \omega_{\mathrm{p}}$ corresponding to a single plasma-dielectric interface. Increasing the central region width is found to introduce a delay in reaching the quasi-static resonance frequencies. The effect of collision is to down shift the mode frequencies for long wavelengths and also to down shift the quasi-static frequencies.
\end{abstract}

\section{Introduction}

Plasma surface waves (PSW) as proper modes of bound plasmas are considered of great importance in different subfields and applications of plasma, such as plasma sources [1], plasma diagnostics [2], plasma produced by lasers, and microelectronics [3]. PSW appear and propagate along the interface separating two different media of positive and negative permittivities, such as the interface between a plasma and a dielectric $[4,5,6]$. The surface wave field decays in both media as it moves inside each medium and away from the interface between them. However, at the interface, the surface wave appears as a periodic surface structure with amplitude reaching a maximum value.

Plasma surface waves were studied intensively in the past decades. The waves were investigated and solutions to the wave equation were obtained for different plasma conditions, such as warm and cold plasmas, magnetized and unmagnetized plasmas $[4,7,8,9,10]$. The electrostatic slow wave solution is valid for phase velocities much below the speed of light, namely, $v_{\phi} \ll c$. In the presence of an external static magnetic field, the spectrum of the surface waves along a plasma-vacuum interface were obtained in different directions [7]. Thermally excited surface plasma wave modes on a metal film surrounded by two identical media have been discussed theoretically and verified experimentally [11, 12].

A detailed study of the modes supported by a metal film sandwiched between dielectrics of relatively high dielectric constants was carried out by S. Sital, et. al., [13]. Antisymmetric and symmetric surface wave modes were found to be supported only when the difference between the dielectric constants was low, while at higher differences, the symmetric bound mode is found to change into a leaky mode.

The electrostatic surface waves of a single collisionless plasma slab, which is bound symmetrically by two dielectrics, were studied by Alsharif et. al. [14]. Surface waves that correspond to symmetric and anti-symmetric modes were observed and the spectra of these modes were shown to be different in the long wavelength limit. Recently, transverse electric waves for a plasma slab sandwiched between two ferrite films have been investigated [15]. The dispersion relation of the surface waves were obtained theoretically, and plots for the effective wave index versus propagation frequency were obtained by numerical calculations in the frequency range where Voigt permeability function has negative values. The results showed two propagation regions around a gap whose width depends on the wave index and the thickness of the plasma slab.

Present article is concerned with the splitting of surface modes supported by the waveguide structure of two identical, parallel, collisional, and homogeneous plasma slabs separated by a central dielectric medium. In Sec.2, the model equations are presented and the spectra of possible surface modes are predicted. Numerical examples of two structures corresponding to vacuumplasma-polyethylene-plasma-vacuum and vacuum-plasmavacuum-plasma-vacuum will be given and discussed in sec.3. Finally we discuss the results and present the main conclusions in Sec.4. 


\section{Model Equations}

We consider a dielectric slab of thickness $d$, that is infinitely extending in the $x y$-plane between two parallel plasma slabs of equal thicknesses $\ell$ and dielectric permittivity $\varepsilon_{\mathrm{p}}$. The rest of the space is taken to be vacuum, as shown in Fig. 1. The wave equation for the electric field $\vec{E}$ in a linear isotropic medium with permittivity $\epsilon(\omega)$ and permeability $\mu_{0}$ in the electrostatic limit is obtained by eliminating the contribution from displacement current. In this case the electric field obeys Poisson's equation.

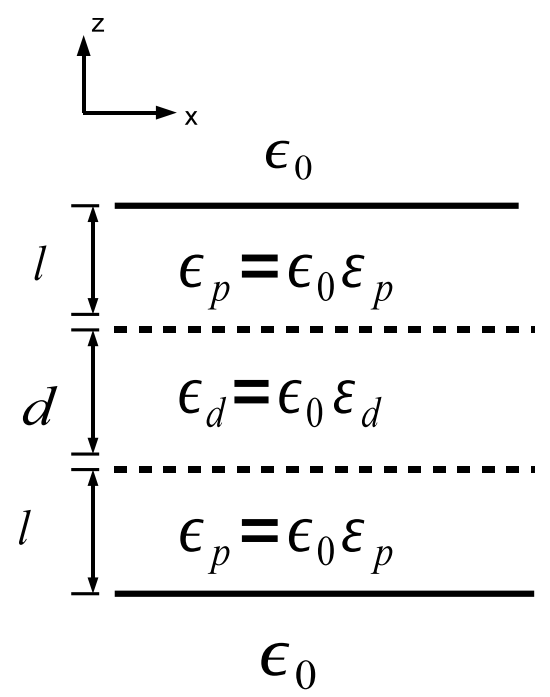

Figure 1: Geometry of the problem.

Without loss of generality, we will consider the surface wave modes that are propagating along the interface in $x$ direction such that

$$
E_{x}(\vec{r})=e_{x}(z) e^{i k x},
$$

where $e_{x}(z)$ is the electric field amplitude, which accounts for the electric field variations with $z$. For unbound slabs in $x$ and $y$, the spatial periodic solution in exponential form with a constant propagation wavenumber $k$ represents a slow wave propagation along $x$ with no reflection. Accordingly, the equation for $e_{x}(z)$ in a homogeneous medium is,

$$
\frac{d^{2} e_{x}(z)}{d z^{2}}-k^{2} e_{x}(z)=0
$$

In the electrostatic limit for propagation along the $x$ axis, the amplitude of the normal electric $e_{z}(z)$ in terms of $e_{x}(z)$ is as given by:

$$
e_{z}(z)=-\frac{i}{k} \frac{d e_{x}(z)}{d z}
$$

From equation (3) we see that it is sufficient to solve for the electric field amplitude $e_{x}(z)$. For the waveguide geometry of Fig.1, the amplitude $e_{x}(z)$ in each slab is,

$$
\begin{array}{cr}
e_{x}^{(1)}=A_{1} e^{k z} & -\infty<z \leq-\ell \\
e_{x}^{(2)}=A_{2} e^{k z}+A_{3} e^{-k z} & -\ell \leq z \leq 0 \\
e_{x}^{(3)}=A_{4} e^{k z}+A_{5} e^{-k z} & 0 \leq z \leq d \\
e_{x}^{(4)}=A_{6} e^{k z}+A_{7} e^{-k z} & d \leq z \leq d+\ell \\
e_{x}^{(5)}=A_{8} e^{-k z} & d+\ell \leq z<\infty
\end{array}
$$

The continuity of the tangential electric field $E_{x}$ and the normal electric displacement $D_{z}$ components at all interfaces involved yields a homogeneous system of eight algebraic equations of the eight unknown integration constants $A_{1}$ thru $A_{8}$. Upon solving the equations of the homogeneous $8 \times 8$ system simultaneously, we obtain the following effective dielectric function, which characterizes all possible electrostatic modes of Fig.1,

$$
\begin{aligned}
& \varepsilon_{\mathrm{p}}^{( \pm)}=-\frac{1}{2}\left(\varepsilon_{\mathrm{d}} \tanh \frac{k d}{2}+1\right) \operatorname{coth} k \ell \pm \\
& \frac{1}{2} \sqrt{\left(\varepsilon_{\mathrm{d}} \tanh \frac{k d}{2}+1\right)^{2} \operatorname{coth}^{2} k \ell-4 \varepsilon_{\mathrm{d}} \tanh \frac{k d}{2}}
\end{aligned}
$$

Assuming the plasma slabs to be homogeneous and warm with a bulk plasma dielectric function that is given by $[4,6,14,16]$,

$$
\varepsilon_{\mathrm{p}}=1-\frac{\omega_{\mathrm{p}}^{2}}{\omega(\omega-j \nu)},
$$

where $\omega_{\mathrm{p}}$ stands for the electron plasma frequency and $\nu$ for an effective collision frequency.

The general expressions for the frequencies of the modes supported by the structure are,

$$
\begin{gathered}
\omega_{1}^{( \pm)}=j \frac{\nu}{2}+\frac{\omega_{\mathrm{p}}}{\sqrt{1-\varepsilon_{\mathrm{p}}^{( \pm)}}} \sqrt{1-\frac{\left(1-\varepsilon_{\mathrm{p}}^{( \pm)}\right) \nu^{2}}{4 \omega_{\mathrm{p}}^{2}}}, \\
\omega_{2}^{( \pm)}=j \frac{\nu}{2}-\frac{\omega_{\mathrm{p}}}{\sqrt{1-\varepsilon_{\mathrm{p}}^{( \pm)}}} \sqrt{1-\frac{\left(1-\varepsilon_{\mathrm{p}}^{( \pm)}\right) \nu^{2}}{4 \omega_{\mathrm{p}}^{2}}}
\end{gathered}
$$

The modes $\omega_{1}^{( \pm)}$and $\omega_{2}^{( \pm)}$of equation (11) and (12) correspond to the surface plasma modes supported by the structure along the plasma-dielectic-plasma interfaces. These modes correspond to a forward and backward propagating wave modes, respectively. For positive frequencies, we will consider only the two modes $\omega_{1}^{(+)}$and $\omega_{1}^{(-)}$. In the next section, the real parts of $\omega_{1}^{(+)}$and $\omega_{1}^{(-)}$will be investigated and the imaginary part will be left out of consideration since it introduces a temporal damping which is independent of the structure parameters. 


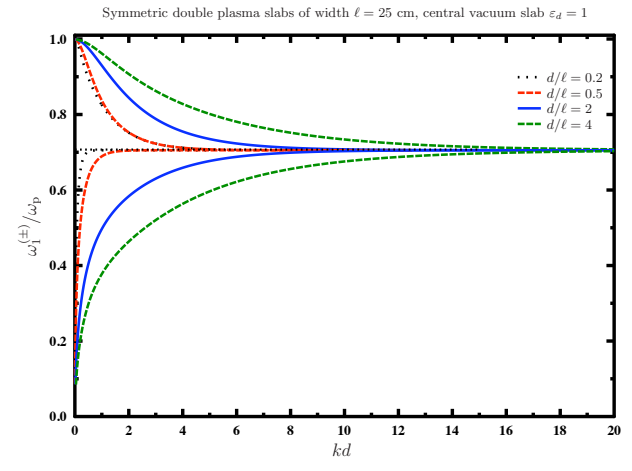

Figure 2: Dispersion curves of two identical plasma slabs with vacuum as a central region.

\section{Numerical Example}

In Fig.2, we use equation (11) to plot $\omega_{1}^{(+)}$and $\omega_{1}^{(-)}$versus $k d=2 \pi d / \lambda$ for different ratios of the central region thickness to the plasma slab thickness $d / \ell$. The curves of Fig.2 show the mode frequencies of vacuum as a central region with $\varepsilon_{d}=1$.

The curves above the quasi-static resonance frequency $\omega=\frac{\omega_{\mathrm{p}}}{\sqrt{1+\varepsilon_{\mathrm{d}}}}=\frac{\omega_{\mathrm{p}}}{\sqrt{2}}=0.707 \omega_{\mathrm{p}}$ are for the mode $\omega_{1}^{(+)}$and those below the quasi-static value are for $\omega_{1}^{(-)}$. For small values of $d / \ell$, the two surface plasmon modes $\omega_{1}^{(+)}$and $\omega_{1}^{(-)}$become coupled at lower values of $k d$ and merge into a single mode of quasi-static frequency $\omega_{1}^{(+)}=\omega_{1}^{(-)}=$ $0.707 \omega_{\mathrm{p}}$. By increasing the width of the central region, which is vacuum in this case, the surface modes are nondegenerate over a wide range of $k d$. For large enough $k d$ values, they again become coupled and then merge into a single mode at $\omega=0.707 \omega_{\mathrm{p}}[4,7,14,16]$.

In Fig.3, we plot $\omega_{1}^{(+)}$and $\omega_{1}^{(-)}$versus $k d$ for different values of $d / \ell$ and for Polyethylene as a central region with a static relative permittivity $\varepsilon_{d}=2.25$ [17]. The curves above the frequency $\omega=\frac{\omega_{\mathrm{p}}}{\sqrt{2}}=0.707 \omega_{\mathrm{p}}$ are for the mode $\omega_{1}^{(+)}$. This plasmon mode starts from the bulk plasma frequency and propagates backward up to the quasi-static resonance frequency $\omega=0.707 \omega_{\mathrm{p}}$ for large $k d$ values. This frequency value corresponds to a surface plasmon frequency of a single plasma-vacuum interface. The curves below the frequency value $\omega=\frac{\omega_{\mathrm{p}}}{\sqrt{3.25}}=0.55 \omega_{\mathrm{p}}$ are for the surface wave mode $\omega_{1}^{(-)}$. This plasmon mode starts from low frequencies near zero and propagates forward until it reaches the frequency $\omega=0.55 \omega_{\mathrm{p}}$ for large $k d$ values. This frequency value corresponds to a surface plasmon quasi-static frequency of a single plasma-dielectric interface.

Two effects can be observed from the curves of Fig.3. First, the surface plasmon modes in the presence of Polyethylene are not coupled over the whole range of $k d$. These modes are propagating independent of each other with distinct quasi-static resonance frequencies. Secondly,

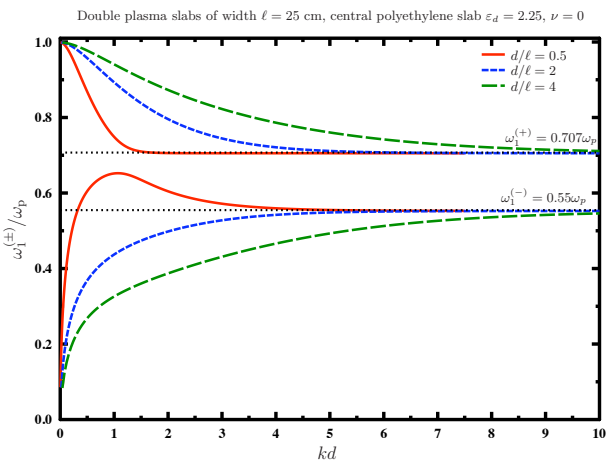

Figure 3: Dispersion curves of two identical plasma slabs with Polyethylene as a central region.

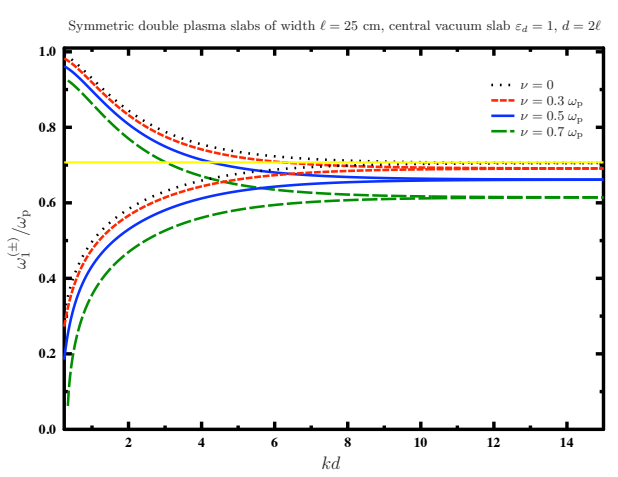

Figure 4: Dispersion curves of two identical plasma slabs with vacuum as a central region..

each plasmon mode reaches its limiting frequency at small $k d$ values for small $d / \ell$, and by increasing the width of Polyethylene slab, the limiting frequencies are reached at higher $k d$ values.

In Fig.4, we use equation (11) to plot the real parts of $\omega_{1}^{(+)}$and $\omega_{1}^{(-)}$versus $k d$ for different collision frequencies $\nu$. The curves of Fig. 4 show the mode frequencies in a collisional plasma with vacuum as a central region between the parallel plasma slabs as a central region $\left(\varepsilon_{d}=1\right)$.

The presence of collisional effect introduces no changes on the coupling of the two surface modes. The effect of collision can be seen as to down shifting the mode frequencies $\omega_{1}^{(+)}$and $\omega_{1}^{(-)}$at very low values of $k d$. For small values of $\nu$, the two surface plasmon modes $\omega_{1}^{(+)}$ and $\omega_{1}^{(-)}$become coupled and merge into a single mode of frequency $\omega_{1}^{(+)}=\omega_{1}^{(-)}=0.707 \omega_{\mathrm{p}}$. By increasing $\nu$, the surface plasmon modes stay coupled and merge into a single mode with an asymptotic frequency below the noncollisional plasma value $\omega=0.707 \omega_{\mathrm{p}}$. For $\nu=0.7 \omega_{\mathrm{p}}$, for example, the modes $\omega_{1}^{(+)}$and $\omega_{1}^{(-)}$are degenerate at frequency $\omega \approx 0.61 \omega_{\mathrm{p}}$. 


\section{Discussion and Conclusions}

In this work, we investigated the coupling of electrostatic surface plasma modes supported by two identical parallel plasma slabs separated by either vacuum or a dielectric (see Fig.1). Upon matching the electrostatic field components at all interfaces involved, we obtained a general characteristic equation for the surface plasma modes. Equation (11) shows the existence of two modes with frequencies $\omega_{1}^{(+)}$ and $\omega_{1}^{(-)}$. In Figs.2-3, we use equation (11) to plot the real parts of $\omega_{1}^{(+)}$and $\omega_{1}^{(-)}$versus $k d=2 \pi d / \lambda$ for different $d / \ell$ and different collision frequencies $\nu$.

Fig. 2 shows the mode frequencies in a plasma with vacuum as a central region $\left(\varepsilon_{d}=1\right)$. At small $k d$ values, the mode $\omega_{1}^{(+)}$starts from the bulk plasma frequency $\omega_{\mathrm{p}}$ and the mode $\omega_{1}^{(-)}$starts from low frequencies near zero. Either for small or large values of $d / \ell$, the two surface plasmon modes $\omega_{1}^{(+)}$and $\omega_{1}^{(-)}$become coupled and merge finally into a single mode of frequency $\omega_{1}^{(+)}=\omega_{1}^{(-)}=$ $0.707 \omega_{\mathrm{p}}$. This corresponds to a surface plasma mode of a single plasma-vacuum interface $[4,6]$. The mode $\omega_{1}^{(+)}$ is a backward wave which tends to quasi-static frequency from above, while $\omega_{1}^{(-)}$is a forward wave that resonates from below.

Fig. 3 shows the real parts of $\omega_{1}^{(+)}$and $\omega_{1}^{(-)}$for different values of $d / \ell$ with Polyethylene as a central region with $\varepsilon_{d}=2.25$ [17]. The plasmon mode $\omega_{1}^{(+)}$starts from the bulk plasma frequency and reaches the surface wave frequency $\omega=0.707 \omega_{\mathrm{p}}$ for large $k d$ values. This asymptotic value corresponds to a surface plasmon frequency of a single plasma-vacuum interface. The plasmon mode $\omega_{1}^{(-)}$ starts from low frequencies near zero and reaches the surface wave frequency $\omega=\frac{\omega_{\mathrm{p}}}{\sqrt{3.25}}=0.55 \omega_{\mathrm{p}}$ for large $k d$ values. This asymptotic value corresponds to a surface plasmon frequency of a single plasma-dielectric interface. The surface plasmon modes in the presence of a Polyethylene central region are decoupled over the whole range of $k d$. These modes $\omega_{1}^{( \pm)}$are propagating independent of each other with distinct asymptotic surface wave frequencies.

The presence of collisional effect introduces no changes on the coupling of the two surface modes. However, the effect is observed to decrease the mode frequencies $\omega_{1}^{(+)}$ and $\omega_{1}^{(-)}$at very low values of $k d$, and accordingly to decrease the quasi-static frequency. For $\nu=0.7 \omega_{\mathrm{p}}$, for example, the modes $\omega_{1}^{(+)}$and $\omega_{1}^{(-)}$are degenerate at frequency $\omega \approx 0.61 \omega_{\mathrm{p}}$.

\section{Acknowledgement}

I E. Yasin and A. Abuzir acknowledge the Deanship of Scientific Research, King Faisal University for their support under grant 1811014.
[1] M. Moisan and Z. Zakrzewski, "Plasma sources based on the propagation of electromagnetic surface waves", J. of Phys. D: App. Phys. 24, pp. 1025-1048 (1991).

[2] G.A. Markov and I.V. Khazanov, "Diagnostics of Plasma Oscillations by the Field of Surface Waves Guided by a Dielectric Waveguide“, Plasma Phys. Rep. 28, pp. 307-318 (2002).

[3] S.A. Magnitskii, V.T. Platonenko, and A. V. Tarasishin, "Surface electromagnetic waves at the interface between vacuum and plasma produced by ultrashort laser pulses“, AIP Conf. Proc. 426, pp. 73-78 (1998).

[4] A. D. Boardman, Electromagnetic Surface Modes (Wiley, New York, 1982).

[5] A.W. Trivelpiece, Slow-Wave Propagation in Plasma Waveguides (San Francisco Press, San Francisco 1967).

[6] Yu. M. Aliev, H. Schlüter, and A. Shivarova, GuidedWave-Produced Plasmas (Springer, Berlin, 2000).

[7] N. Z. Abdel-Shahid and V. I. Pakhomov, “ On the study of the potential surface waves in plasma in the presence of an external magnetic field“", Plasma Phys. 12, pp. 55-65 (1970).

[8] A. Shivarova and I. Zhelyazkov, " Surface waves in a homogeneous plasma sharply bounded by a dielectric“, Plasma Phys. 20, pp. 1049-1073 (1978).

[9] H. J. Lee, " Electrostatic surface waves in a magnetized two-fluid plasma“, Plasma Phys. Controlled Fusion 37, pp. 755-762 (1995).

[10] H. J. Lee and Sang-Hoon Cho, “ Dispersion of surface waves propagating along planar interfaces between plasmas or plasma and free space“, Plasma Phys. Controlled Fusion 37, pp. 989-1002 (1995).

[11] H. Boersch, J. Geiger, A. Imbusch, and N. Niedrig, “ High resolution investigation of the energy losses of $30 \mathrm{keV}$ electrons in aluminum foils of various thicknesses“, Phys. Lett. 22, pp. 146-147 (1966).

[12] J. B. Swan, A. Otto, and H. Fellenzer, “ Observed retardation effects on the energy of the w+-surface plasmons in thin aluminum foils“, Phys. Status Solidi 23, pp. 171-176 (1967).

[13] S. Sital and E. Sharma, “ Surface plasmon modes of dielectric-metal-dielectric waveguides and applications", Journal of Electronics and Communication Engineering Volume 12, Issue 2, Ver. I, PP 08-19 (2017).

[14] A.I. Al-Sharif, A.M. Al-Khateeb, S.H. Mahmood, N.M. Laham, and M.S. Bawa'aneh " Coupling of slow phase velocity surface waves of planar plasma bounded by two dielectrics", The Arabian Journal for Science and Engineering, Volume 32, Number 1A.

\section{References}


[15] R. Ali, B. Zamir, H.A. Shah" Transverse electric surface waves in a plasma medium bounded by magnetic materials', Results in Physics 8 (2018) 243-248.

[16] K. L. Kliewer and R. Fuchs, " Theory of dynamical properties of dielectric surfaces", Adv. Chem. Phys. 27, pp. 355-541 (1974).

[17] D. L. Chung, Functional Materials: Electrical, Dielectric, Electromagnetic, Optical and Magnetic Applications, World Scientific, 2010. 